\title{
The Applicability of Mouse Models to the Study of Human Disease
}

\author{
Kristina Rydell-Törmänen and Jill R. Johnson
}

\begin{abstract}
The laboratory mouse Mus musculus has long been used as a model organism to test hypotheses and treatments related to understanding the mechanisms of disease in humans; however, for these experiments to be relevant, it is important to know the complex ways in which mice are similar to humans and, crucially, the ways in which they differ. In this chapter, an in-depth analysis of these similarities and differences is provided to allow researchers to use mouse models of human disease and primary cells derived from these animal models under the most appropriate and meaningful conditions.

Although there are considerable differences between mice and humans, particularly regarding genetics, physiology, and immunology, a more thorough understanding of these differences and their effects on the function of the whole organism will provide deeper insights into relevant disease mechanisms and potential drug targets for further clinical investigation. Using specific examples of mouse models of human lung disease, i.e., asthma, chronic obstructive pulmonary disease, and pulmonary fibrosis, this chapter explores the most salient features of mouse models of human disease and provides a full assessment of the advantages and limitations of these models, focusing on the relevance of disease induction and their ability to replicate critical features of human disease pathophysiology and response to treatment. The chapter concludes with a discussion on the future of using mice in medical research with regard to ethical and technological considerations.
\end{abstract}

Key words Mouse, Model, Disease, Genetics, Physiology, Immunology, Ethics

\section{The Mouse: From Pest, to Pet, to Predominant Tool in Medical Research}

Although the genetic lineages of mice and humans diverged around 75 million years ago, these two species have evolved to live together, particularly since the development of agriculture. For millennia, mice (Mus musculus) were considered to be pests due to their propensity to ravenously consume stored foodstuff ( $m u s h$ in ancient Sanskrit means "to steal" [1]) and their ability to adapt to a wide range of environmental conditions. Since the 1700s, domesticated mice have been bred and kept as companion animals, and in Victorian England, "fancy" mice were prized for their variations in coat color and comportment; these mouse strains were the 
forerunners to the strains used in the laboratory today. Robert Hooke performed the first recorded inquiry-driven experiments on mice in 1664, when he investigated the effects of changes in air pressure on respiratory function [2]. More recently, with data from the Human Genome Project and sequencing of the Mus musculus genome showing remarkable genetic homology between these species, as well as the advent of biotechnology and the development of myriad knockout and transgenic mouse strains, it is clear why the mouse has become the most ubiquitous model organism used to study human disease. In addition, their small size, rapid breeding, and ease of handling are all important advantages to scientists for practical and financial reasons. However, keeping in mind that mice are fellow vertebrates and mammals, there are ethical issues inherent to using these animals in medical research. This chapter will provide an overview of the important similarities and differences between Mus musculus and Homo sapiens and their relevance to the use of the mouse as a model organism and provide specific examples of the quality of mouse models used to investigate the mechanisms, pathology, and treatment of human lung diseases. We will then conclude with an assessment of the future of mice in medical research considering ethical and technological advances.

As a model organism used to test hypotheses and treatments related to human disease, it is important to understand the complex ways in which mice are similar to humans, and crucially, the ways in which they differ. A clear understanding of these aspects will allow researchers to use mouse models of human disease and primary cells derived from mice under the most appropriate and meaningful conditions.

\section{Applicability of Mouse Models to Human Disease}

\subsection{Genetics}

In 2014, the Encyclopedia of DNA Elements (ENCODE) program published a comparative analysis of the genomes of Homo sapiens and Mus musculus [3], as well as an in-depth analysis of the differences in the regulatory landscape of the genomes of these species [4]. ENCODE, a follow-up to the Human Genome Project, was implemented by the National Human Genome Research Institute (NHGRI) at the National Institutes of Health in order to develop a comprehensive catalog of protein-encoding and nonproteincoding genes and the regulatory elements that control gene expression in a number of species. This was achieved using a number of genomic approaches (e.g., RNA-seq, DNase-seq, and ChIP-seq) to assess gene expression in over 100 mouse cell types and tissues; the data were then compared with the human genome.

Overall, these studies showed that although gene expression is fairly similar between mice and humans, considerable differences were observed in the regulatory networks controlling the activity of 
the immune system, metabolic functions, and responses to stress, all of which have important implications when using mice to model human disease. In essence, mice and humans demonstrate genetic similarity with regulatory divergence. Specifically, there is a high degree of similarity in transcription factor networks but a great deal of divergence in the cis-regulatory elements that control gene transcription in the mouse and human genomes. Moreover, the chromatin landscape in cell types of similar lineages in mouse and human is both developmentally stable and evolutionarily conserved [3]. Of particular relevance regarding modeling human diseases involving the immune system, in its assessment of transcription factor networks, the Mouse ENCODE Consortium revealed potentially important differences in the activity of ETS1 in the mouse and human genome. Although conserved between the two species, divergence in ETS1 regulation may be responsible for discrepancies in the function of the immune system in mouse and human [4]. Certainly, the biological consequences of these differences in gene expression and regulation between human and mouse invite further investigation.

2.2 Anatomy and Physiology
The anatomical and physiological differences between model organisms and humans can have profound impacts on interpreting experimental results. Virtually every biological process under investigation in experimental studies involves at least one anatomical structure. To aid in interpretation, many anatomy compendia have been developed for model organisms; the most useful organize anatomical entities into hierarchies representing the structure of the human body, e.g., the Foundational Model of Anatomy developed by the Structural Informatics Group at the University of Washington [5]. Although an analysis of the myriad differences between mouse and human anatomy is beyond the scope of this chapter, a few of the most critical issues that have an impact on the interpretation of data from mouse experiments should be mentioned.

The most obvious difference between mice and humans is size; the human body is about 2500 times larger than that of the mouse. Size influences many aspects of biology, particularly the metabolic rate, which is correlated to body size in placental mammals through the relationship $\mathrm{BMR}=70 \times$ mass $(0.75)$, where $\mathrm{BMR}$ is the basal metabolic rate (in $\mathrm{kcal} / \mathrm{day}$ ). Thus, the mouse BMR is roughly seven times faster than that of an average-sized human [6]. This higher BMR has effects on thermoregulation, nutrient demand, and nutrient supply. As such, mice have greater amounts of metabolically active tissues (e.g., liver and kidney) and more extensive deposits of brown fat [6]. Furthermore, mice more readily produce reactive oxygen species than do humans, which is an important consideration when modeling human diseases involving the 
induction of oxidative stress (i.e., aging, inflammation, and neurodegeneration) [6].

The lung provides an excellent example of the similarities and differences between human and mouse anatomy. Similar to the human organ, the mouse lung is subdivided into lobes of lung parenchyma containing a branching bronchial tree and is vascularized by the pulmonary circulation originating from the right ventricle. There are a number of subtle variations in this general structure between species, i.e., the number of lobes on the right and left, the branching pattern, and the distribution of cartilage rings around the large airways, but the most important differences between the mouse and human lung are related to the organism's size (airway diameter and alveolar size are naturally much smaller in the mouse) and respiratory rate. Moreover, there are important differences in the blood supply of the large airways in humans versus mice [7]. Specifically, the bronchial circulation (a branch of the high-pressure systemic circulation that arises from the aorta and intercostal arteries) supplies a miniscule proportion of the pulmonary tissue in mice (the trachea and bronchi) compared to humans; the majority of the lung parenchyma is supplied by the low-pressure, high-flow pulmonary circulation. In the mouse, these systemic blood vessels do not penetrate into the intraparenchymal airways, as they do in larger species [8]. This difference, although subtle, has important ramifications regarding the vascular supply of lung tumors which, in humans, is primarily derived from the systemic circulation [9]. These differences may also have profound consequences when modeling human diseases involving the lung vasculature.

\subsection{Immunology}

The adaptive immune system evolved in jawed fish about 500 million years ago, well before the evolution of mammals and the divergence of mouse and human ancestral species [10]. Many features of the adaptive immune system, including antigen recognition, clonal selection, antibody production, and immunological tolerance, have been maintained since they first arose in early vertebrates. However, the finer details of the mouse and human immune systems differ considerably, which is not surprising since these species diverged 75 million years ago [6]. While some have claimed that these differences mean that research into immunological phenomena in mice is not transferable to humans, as long as these differences are understood and acknowledged, the study of mouse immune responses can continue to be relevant.

Research on mice has been vital to the discovery of key features of both innate and adaptive immune responses; for example, the first descriptions of the major histocompatibility complex, the T cell receptor, and antibody synthesis were derived from experiments performed on mice [6]. The general structure of the immune system is similar in mice and humans, with similar mediators and 
Table 1

A brief overview of the immunological differences between mice and humans

\begin{tabular}{|c|c|c|c|}
\hline Attribute & Mouse & Human & References \\
\hline $\begin{array}{l}\text { Proportion of } \\
\text { leukocytes in } \\
\text { the blood }\end{array}$ & $\begin{array}{l}75-90 \% \text { lymphocytes } \\
10-25 \% \text { neutrophils }\end{array}$ & $\begin{array}{l}50-70 \% \text { neutrophils } \\
30-50 \% \text { lymphocytes }\end{array}$ & [13] \\
\hline $\begin{array}{l}\text { Antigen } \\
\text { presentation }\end{array}$ & $\begin{array}{l}\text { Endothelial cells do not express } \\
\text { MHC Class II, cannot activate } \\
\text { CD4+ T cells }\end{array}$ & $\begin{array}{l}\text { Endothelial cells express MHC } \\
\text { Class II and present antigen to } \\
\text { CD4+ T cells }\end{array}$ & [14] \\
\hline $\begin{array}{l}\text { Costimulatory } \\
\text { signaling }\end{array}$ & $\begin{array}{l}80 \% \text { of CD } 4+\text { and } 50 \% \text { of } \mathrm{CD} 8+ \\
\text { T cells express CD28 } \\
\text { ICOS is not required for B cell } \\
\text { maturation } \\
\text { B7-H3 inhibits T cell activation }\end{array}$ & $\begin{array}{l}100 \% \text { of } \mathrm{CD} 4+\text { and } \mathrm{CD} 8+\mathrm{T} \text { cells } \\
\text { express CD } 28 \\
\text { ICOS is required for B cell } \\
\text { maturation and IgM production } \\
\text { B7-H3 promotes T cell activation }\end{array}$ & $\begin{array}{l}{[12]} \\
{[15,16]} \\
{[17]}\end{array}$ \\
\hline $\begin{array}{l}\text { Immunoglobulin } \\
\text { isotypes }\end{array}$ & $\begin{array}{l}\operatorname{IgD}, \operatorname{IgM}, \operatorname{IgA}, \operatorname{IgE}, \operatorname{IgGl}, \\
\operatorname{IgG} 2 \mathrm{a} / \mathrm{c}, \operatorname{IgG} 2 \mathrm{~b}, \operatorname{IgG} 3\end{array}$ & $\begin{array}{l}\operatorname{IgD}, \operatorname{IgM}, \operatorname{IgAl}, \operatorname{IgA} 2, \operatorname{IgE}, \operatorname{IgGl}, \\
\text { IgG2, IgG3, IgG4 }\end{array}$ & [12] \\
\hline $\begin{array}{r}\text { Immunoglobulin } \\
\text { class switching }\end{array}$ & IL-4 induces IgGl and $\operatorname{IgE}$ & IL-4 induces IgG4 and IgE & [18] \\
\hline $\begin{array}{l}\text { Helper } \mathrm{T} \text { cell } \\
\quad \text { differentiation }\end{array}$ & $\begin{array}{l}\text { IFN- } \alpha \text { does not activate STAT4 } \\
\text { and does not induce Th1 } \\
\text { polarization } \\
\text { Clear Thl/Th2 differentiation in } \\
\text { mice }\end{array}$ & $\begin{array}{l}\text { IFN- } \alpha \text { induces Thl polarization via } \\
\text { STAT4 } \\
\text { Multiple T helper cell subsets occur } \\
\text { simultaneously }\end{array}$ & {$[20]$} \\
\hline $\begin{array}{l}\text { Responses to } \\
\text { infection }\end{array}$ & $\begin{array}{l}\text { Eradication of schistosomiasis } \\
\text { requires a Thl response and } \\
\text { IFN- } \gamma \\
\text { Low susceptibility to } \\
\quad \text { Mycobacterium tuberculosis; } \\
\text { noncaseating granulomas; no } \\
\text { latent infection }\end{array}$ & $\begin{array}{l}\text { Eradication of schistosomiasis } \\
\text { requires a Th2 response and } \operatorname{IgE} \\
\text { Highly susceptible to } \\
\text { Mycobacterium tuberculosis; } \\
\text { caseating granulomas; latent } \\
\text { infection is common }\end{array}$ & {$[22]$} \\
\hline
\end{tabular}

cell types involved in rapid, innate immune responses (complement, macrophages, neutrophils, and natural killer cells) as well as adaptive immune responses informed by antigen-presenting dendritic cells and executed by B and T cells. However, due to the anatomical and physiological differences between these species as described above, divergence in key features of the immune system, such as the maintenance of memory $\mathrm{T}$ cells (related to the life span of the organism) and the commensal microbiota (related to the lifestyle of the organism), has arisen [11].

Similar to what has been discovered regarding the genetics of mice and humans, i.e., broad similarities in structure but considerable differences in regulation, there are a number of known discrepancies in the regulation of innate and adaptive immunity in 
mice versus humans, including the balance of leukocyte subsets, $\mathrm{T}$ cell activation and costimulation, antibody subtypes and cellular responses to antibody, Thl/Th2 differentiation, and responses to pathogens (described in detail in Table 1). In addition to these differences in immune cell functions, the expression of specific genes involved in immune responses also differs, particularly those for Toll-like receptors, defensins, NK inhibitory receptors, Thy-1, and many components of chemokine and cytokine signaling; additionally, differences between mouse strains are known to exist for many of these mediators [12].

Another important consideration when using mice to perform immunological research (with a view to translating these findings to human medicine) is the availability of hundreds of strains of genetically modified mice that have enabled exquisitely detailed studies on immune cell function, regulation, and trafficking. Many of these strains involve the expression of inducible Cre or Cas9 that allow for targeted knockdown or overexpression of key immune function-related genes in specific cell types at specific moments in time. However, it is important to note that drift between mouse colonies has long been known to occur. In fact, a recent report described the fortuitous discovery of a point mutation in the natural cytotoxicity receptor 1 (NCRl) gene in the C57/Bl6 CD45.1 mouse strain, resulting in absent NCRI expression. This mutation was found to have profound effects on the response of mice to viral infection, i.e., the mice were resistant to cytomegalovirus infection but more susceptible to influenza virus [23]. This cautionary tale highlights the importance of understanding the genetic evolution of laboratory strains of mice, the effect of these genetic and immunological changes on mouse biology, and the impact on the translation of these results to human medicine.

In addition to the differences between mouse and human genetics, physiology, and immunology highlighted above, several factors must also be taken into account when performing in vitro assays using isolated mouse cells and applying these findings to our understanding of human disease. Particularly with regard to stem cell research, it should be noted that the telomeres of mouse cells are five- to tenfold longer than human telomeres, resulting in greater replicative capacity [24]. There are also important differences in the regulation of pluripotency and stem cell differentiation pathways in humans and mice [25]. Moreover, there are considerable species differences in the longevity of cultured cells; for example, mouse fibroblasts are capable of spontaneous immortalization in vitro, whereas human fibroblasts become senescent and ultimately fail to thrive in culture [26].

In summary, although there are considerable differences between mice and humans, constant improvement in the analytical techniques used to delineate these differences and their effects on whole organism and cell function have provided vital information 
and contributed to our understanding of both murine and human biology. Experimentation employing mouse models of human disease will continue to provide key insights into relevant disease mechanisms and potential drug targets for further clinical investigation. However, several important considerations must be taken into account when selecting a mouse model of human disease, as described in the following section, using mouse models of human lung disease to illustrate this point.

\section{Mouse Models of Human Disease}

The two most salient features of a mouse model of human disease are the accuracy of its etiology (it employs a physiologically relevant method of disease induction) and its presentation (its ability to recapitulate the features of human disease). The relevance of any given mouse model can be judged on the basis of these two criteria, and there is considerable variation within mouse models of human disease in this regard. As a full assessment of the advantages and limitations of all currently available mouse models of human disease would be prohibitively long and complex, here we have elected to assess the accuracy of currently available models of human lung diseases, i.e., asthma, chronic obstructive pulmonary disease, and pulmonary fibrosis, focusing on the relevance of disease induction in these models and their ability to replicate critical features of human disease pathophysiology and response to treatment.

The first and foremost notion when modeling human disease in mice is to acknowledge the species differences, which are significant [27]. As described above, genetics, anatomy, physiology, and immunology differ between mice and humans, but despite these differences, mouse models of human disease are useful and necessary, as long as data interpretation is performed appropriately.

\section{Asthma}

An elegant example of differences between mice and humans that must be considered when designing a mouse model of human inflammatory lung disease is the key effector cell type in human asthma, i.e., mast cells. These leukocytes differ in granule composition as well as localization in the mouse and human airways [28]. Mice mostly lack mast cells in the peripheral lung [29], whereas humans have numerous mast cells of multiple subpopulations in the alveolar parenchyma [30]. Another example is anatomy: in contrast to humans, mice lack an extensive pulmonary circulation, which may have significant effects on leukocyte adhesion and migration, and subsequently inflammation [31]. Still, as long as these differences are taken into consideration, mouse models can be 
powerful tools in the discovery and exploitation of new targets for the treatment of human disease.

The World Health Organization (WHO) defines asthma as a chronic disease characterized by recurrent attacks of breathlessness and wheezing, which may vary in severity and frequency from person to person. The disease is characterized by airway hyperresponsiveness, airway smooth muscle thickening, increased mucus secretion and collagen deposition, as well as prominent inflammation affecting both large and small airways [32]. Nowadays, it is recognized that asthma is not a single homogenous disease but rather several different phenotypes united by similar clinical symptoms $[32,33]$.

Only a few animal species develop asthma naturally, including cats and horses [34, 35], whereas mice do not [31]. However, mice can be manipulated to develop a type of allergic airway inflammation, which is similar in many ways to the human disease, in response to different aeroallergens [36]. Importantly, these models are capable of recapitulating only the allergic type of human asthma and have less relevance for other types of asthma (i.e., endotypes induced by medication, obesity, and air pollution).

As with many human diseases, asthma has a complex and multifaceted etiology, where environmental factors, genetic susceptibility, and microbial colonization all contribute; thus, it is important to take strain differences into consideration. Generations of inbreeding have created mouse strains that differ not only in coat color and disposition but also from a physiological, immunological, and genetic perspective. Different strains may be more susceptible to allergic airway inflammation or pulmonary fibrosis, whereas others are more or less resistant. Choosing the right strain to model a specific disease or pathologic event is thus essential. The most widely used strains for models of allergic airway inflammation are $\mathrm{BALB} / \mathrm{c}$ and $\mathrm{C} 57 \mathrm{BL} / 6$. These strains differ regarding the type of immune response mounted to an inhaled allergen: $\mathrm{C} 57 \mathrm{BL} / 6$ is generally considered a $\mathrm{T}_{\mathrm{H}} \mathrm{l}$-skewed strain, whereas $\mathrm{BALB} / \mathrm{c}$ is regarded as a $\mathrm{T}_{\mathrm{H}} 2$-skewed strain [36]. Due to their strong $\mathrm{T}_{\mathrm{H}} 2$ response, and subsequent development of robust asthmatic responses, $\mathrm{BALB} / \mathrm{c}$ has been commonly used to model asthma [37]. However, most humans do not express such a strongly $\mathrm{T}_{\mathrm{H}} 2$-skewed immune system, suggesting this strain may not be the best model of human disease; instead, C57BL/6 may be more suitable as immune responses in this strain are more similar to those of atopic human subjects [37]. Furthermore, as C57BL/6 is the most commonly used strain for the development of genetically manipulated mice, using these mice allows for very specific investigations into disease pathology; thus, this strain is increasingly used in models of human lung disease. 
4.1 Ovalbumin

4.2 House Dust Mite
Besides the genetic differences in the mouse strains used in these models, the etiology (the method of disease induction) of commonly used models of asthma is highly variable. In humans with allergic asthma, environmental allergen exposure occurs at the airway mucosa; the immune response is coordinated in the bronchopulmonary lymph nodes, and the T cells, macrophages, and eosinophils recruited as part of this response travel to the lung where they mediate the cardinal features of asthma: airway inflammation, structural remodeling of the airway wall, and airway hyperreactivity [38]. Ideally, these features should be found in a physiologically relevant mouse model of asthma. However, for the sake of cost and convenience, early mouse models of asthma used the surrogate protein ovalbumin (OVA) [31] rather than an environmental allergen to induce an immune response, which also requires the use of a powerful $\mathrm{T}_{\mathrm{H}} 2$-polarizing adjuvant such as alum delivered via the intraperitoneal route, followed by OVA nebulization-a clear divergence from the etiology of human asthma [36]. In terms of disease presentation, mice develop some hallmarks of asthma, including airway eosinophilic inflammation, goblet cell metaplasia, and increased airway smooth muscle density [31]. After the cessation of OVA exposure, most of the remodeling resolves, although some structural alterations remain up to 1 month after the last challenge [39]. Based on these attributes, the OVA model is primarily a model to investigate the initiation of inflammation, rather than the chronic progression and maintenance of inflammation [31].

A clear advantage with the OVA model is the number of studies where it is used; both the pros and cons are familiar. It is easy to find a suitable protocol, and the model is readily accessible and flexible regarding the number of sensitizations and allergen doses. The model is relatively easy to reproduce, as OVA and different adjuvants are easily obtained. However, the resolution of remodeling following the cessation of allergen provocations is a disadvantage, as is the practical problem with the nebulization of an allergen - it ends up in the mouse's coat and is ingested during grooming, potentially resulting in systemic exposure (this is particularly relevant in models employing systemic, intraperitoneal sensitization). In addition, concerns have been raised against the use of adjuvants to induce the immunological response, as well as the clinical relevance of OVA as an allergen, which have driven the development of more clinically relevant allergens and models [31].

The common environmental aeroallergen house dust mite (HDM) extract is increasingly used to initiate disease in mouse models of allergic airway inflammation, as it is a common human allergen (around 50\% of asthmatics are sensitized to HDM [40]) that evokes asthma attacks and other allergic responses in susceptible individuals. In addition, HDM has inherent allergenic properties, likely 
4.3 Cockroach, Aspergillus, and Other Model Allergens due to components with protease activity [40], so there is no need to use an adjuvant, thus improving the etiological similarity of these models with the clinical situation [41]. In contrast to OVA, prolonged exposure of HDM (up to 7 weeks) induces asthma-like severe airway inflammation with prominent eosinophilia, severe hyperreactivity to methacholine, and robust remodeling of the airway wall [41], i.e., the presentation of chronic respiratory HDM exposure in mice effectively recapitulates the key features of human allergic asthma. Importantly, the airway structural changes induced by chronic HDM exposure, such as increased collagen deposition, airway smooth muscle thickening, and microvascular alterations, persist for at least 4 weeks after the cessation of HDM exposure [42], another commonality with human asthma in which airway remodeling is currently considered to be irreversible.

Thus, the advantages of using HDM as the allergen in mouse models of asthma are the clinical relevance of the allergen [43] and the route of delivery via the respiratory tract. Moreover, studies have shown that the type of inflammation and characteristics of tissue remodeling are relatively similar to those seen in human asthmatics $[35,41,43]$. One disadvantage is the complexity of HDM extract; as a consequence of this complexity, variations exist in some components between batches, particularly regarding the content of lipopolysaccharide, so reproducibility in these studies may be problematic.

With similarity to HDM, these models were developed to be as clinically relevant as possible, as many patients suffer from allergy toward cockroach allergen, molds, and other environmental irritants. A common feature of these allergens is their complex nature, as they commonly consist of a mix of different allergic epitopes and fragments. This complexity is most likely why the immunological reaction in mice is relatively similar to that seen in asthmatics [44].

Cockroach allergen (CRA) is a common allergen, known to induce asthma in susceptible individuals; thus, it shares with HDM the advantage of being highly clinically relevant [45]. CRA induces peribronchial inflammation with significant eosinophilic inflammation and transient airway hyperresponsiveness, both of which can be increased by repeated administrations of the allergen [45].

Colonization of the airways with Aspergillus fumigatus is the cause of allergic bronchopulmonary aspergillosis (ABPA), a disease where the lungs are colonized by the fungus, but allergens from Aspergillus fumigatus can also induce asthma similar to other allergens [46]. The reaction to Aspergillus allergens is robust, and often no adjuvants are needed to elicit inflammation [46]. In addition to Aspergillus, other fungi such as Penicillium and Alternaria can also induce asthma in humans and have been used to model disease in mice [47]. A common difficulty with these allergens is the method of administration, as the physiological route is believed to be the 
inhalation of dry allergens; mimicking this route with a nebulizer introduces the risk of the animals ingesting the allergen and thus causing systemic responses [47].

4.4 Modeling Asthma Exacerbations
Exacerbations of asthma are defined as the worsening of symptoms, prompting an adjustment in treatment, and are believed to be associated with increased inflammation in the distal airways. Clinically, exacerbations are believed to be induced by infections (most common), allergen exposure, or pollutants, which can be modeled in different ways $[48,49]$ :

1. Infections with viruses and bacteria or exposure to proteins/ DNA/RNA derived from these microbes.

2. Administration of a high dose of allergen in a previously sensitized animal.

3. Exposure to environmental pollutants, such as diesel exhaust or ozone.

Modeling exacerbations adds a layer of complexity, as robust ongoing allergic airway inflammation needs to be established first, before challenge with the exacerbating agent. Both the OVA and HDM models are used in this respect, and in both cases chronic protocols extending for several weeks before triggering an exacerbation have been used [48].

\section{Chronic Obstructive Pulmonary Disease}

Chronic obstructive pulmonary disease (COPD) is characterized by chronic airway obstruction, in contrast to asthma where the obstruction is reversible (particularly in response to bronchodilator treatment). Clinically, in COPD, chronic bronchitis and emphysema can occur either separately or in combination. COPD is almost always associated with either first- or secondhand tobacco smoking or in rare cases with a deficiency in the production of $\alpha \mathrm{l}$ antitrypsin (a serpin that prevents elastin breakdown as a result of neutrophil degranulation) [50]. The etiology of COPD is highly complex and is believed to develop after many years of smoking in combination with other known factors such as genetic susceptibility or environmental factors [51]. In similarity to asthma, inflammation is a major component in COPD, but the leukocyte profile is very different: the most prominent players in COPD-related inflammation are neutrophils and, to some degree, macrophages [51]. Due to the complex etiology of COPD, it is difficult to recapitulate all aspects of this disease in a single model, so in most cases, the aim is to induce COPD-like lesions by exposing mice to tissue-damaging substances (usually cigarette smoke) or to mimic emphysema by the administration of tissue-degrading enzymes $[27,51]$. 
5.1 Cigarette Smoke

5.2 Protease Instillation
Clearly, mice do not smoke cigarettes on their own, so to model COPD by cigarette smoke (CS) inhalation, the mice need to be exposed to unfiltered CS in an induction chamber; moreover, in an attempt to better model the chronic aspects of COPD, this needs to be performed for a prolonged period of time. Mice are very tolerant to CS, but eventually (over a period of several weeks), CS induces pulmonary neutrophilic inflammation that is associated with some degree of tissue degradation and destruction [51]. An important advantage of this model is the fact that CS is the actual irritant responsible for disease in humans, and mice develop several features similar to the clinical disease, making this model highly clinically relevant [27]. A significant drawback is the self-limitation of the model-the pathological changes do not progress after the cessation of CS exposure [51]. Furthermore, the exposure time needed for mice to develop COPD-like pathology is extensive, i.e., studies have shown that an exposure protocol of 5 days per week for a minimum of 3 months is needed to generate robust structural changes to the lung [52]. The pathological image in COPD is complex and varies greatly between patients, commonly encompassing chronic bronchitis and bronchiolitis, emphysema, fibrosis, and airway obstruction. Although mice develop some of these symptoms when exposed to CS, they do not develop all the symptoms of human disease; thus, CS has advantages as a model but fails to mimic the complexity of the clinical situation and disease presentation [27].

Other models of COPD rely on the administration of proteases (protein-degrading enzymes) that are believed to be involved in the pathology of this disease in a subset of patients, such as elastindegrading elastase. This approach mimics the emphysematous changes seen in COPD, but the pathological process underlying tissue destruction is likely very different compared to the clinical situation [51], as very few patients show evidence of elastase dysregulation [27]. However, if the aim of the study is to investigate the general effect of protease-induced tissue destruction and regeneration, then this is a highly relevant method [51]. Some studies on COPD have also used genetically modified animals, such as mice overexpressing collagenase, which results in tissue destruction without inflammation or fibrosis with an end result fairly similar to the type of emphysema observed in COPD [53].

\section{Pulmonary Fibrosis}

Pulmonary fibrosis, the accumulation of fibrotic tissue within the alveolar parenchyma, is merely a symptom of disease, and the etiology of this pathology in humans varies greatly [54]. The 
most enigmatic class is perhaps the idiopathic interstitial pneumonias, especially idiopathic pulmonary fibrosis (IPF). IPF is a debilitating and progressive disease with a grave prognosis, characterized by progressive fibrosis believed to reflect aberrant tissue regeneration [55]. As the reason behind this defective repair is unknown, although a combination of immunological, genetic, and environmental factors are suspected, it is very difficult to model disease in a clinically relevant fashion [56]. The most common method used to model pulmonary fibrosis in mice is administration of the chemotherapeutic agent bleomycin; this agent is known to cause pulmonary fibrosis in humans as well, but this may not accurately reflect the true etiology of most cases of human disease. The strain of choice is $\mathrm{C} 57 \mathrm{BL} / 6$, as it is prone to developing pulmonary fibrosis, whereas $\mathrm{BALB} / \mathrm{c}$ is relatively resistant, a feature believed to reflect the cytokine response following cellular stress and damage [57]. Bleomycin administration can be performed locally or systemically, producing very different results.

6.1 Local Bleomycin Administration
The most common model of pulmonary fibrosis is a single intranasal or intratracheal administration of bleomycin, with analysis 3 to 4 weeks later. During this time, the drug causes acute tissue damage in a restricted area of the lung (where the solution ends up during administration), followed by intense inflammation in this area and subsequent fibrosis, which gradually resolves within weeks. However, if older mice are used, the fibrosis will persist longer than in younger mice, which is in accordance with clinical IPF, where the majority of the patients are 65 years of age or older $[56,58]$.

A great advantage of this model is how well-characterized it is. In addition, local administration is labor-effective, as only one administration is required and the result is highly reproducible. The fibrosis is robust, only affects the lungs, and the accumulation of extracellular matrix can be easily measured using standard techniques [58]. Furthermore, as it is used throughout the world, studies performed in different labs and by different groups can be compared relatively easily. Unfortunately, the intense pulmonary inflammation may be lethal, and fatalities are to be expected with this model [59], representing an important ethical limitation. Furthermore, fibrosis is heterogeneous-it develops where the bleomycin solution is deposited. The solution usually deposits within the central lung, a localization that is not in agreement with the clinical situation where fibrosis is located in the more distal regions of the lung parenchyma. In addition, the fibrosis that develops as a result of severe tissue damage is self-limiting and reversible, unlike what is observed clinically [58]. The severe degree of tissue damage induced by bleomycin may in fact be more relevant for modeling acute lung injury (ALI) or acute respiratory distress syndrome (ARDS). 


\subsection{Systemic Bleomycin Administration}

6.3 Fluorescein Isothiocyanate Administration

\subsection{TGF- $\beta$} Overexpression
Bleomycin can also be administered systemically, through intravenous or subcutaneous injection. In contrast to local administration, this route requires multiple administrations and is thus more laborintensive [58]. Some studies have described the usage of osmotic mini-pumps, where bleomycin is slowly administered over a short period of time, and then fibrosis continues to develop over subsequent weeks [60]. Irrespective of the route of delivery, systemic administration results in more homogenous fibrosis, affecting the entire lung through the pulmonary endothelium and persisting much longer than following local administration [61]. The main advantages of systemic administration are that inflammation is limited, while the fibrosis is more apparent and displays a more distal pattern, all of which mimics the clinical situation relatively well. The multiple administrations allow for lower doses with each injection; this is less stressful to the animals and results in little to no mortality [61] and is thus more ethically acceptable. A major disadvantage with this model is that it takes time for fibrosis to develop [58], which may be the reason it is used relatively scarcely, and thus the pathological development is less well-understood. In addition, as IPF is a local disease, local administration of the etiologic agent may better mimic the clinical reality [56].

The administration of fluorescein isothiocyanate (FITC) induces focal inflammation, primarily involving mononuclear cells and neutrophils, and localizes in areas where the FITC solution is deposited [58]. Antibodies against FITC can be detected after 1 week, and the fibrosis persists for up to 5 months after instillation [58]. The benefits of this model are mainly related to the persistent fibrosis that does not appear to be self-limiting, thus reflecting the clinical situation, and it is also very easy to determine which part of the lung has been exposed to FITC, as the molecule is fluorescent [58]. It is also an advantage that both $\mathrm{C} 57 \mathrm{BL} / 6$ and $\mathrm{BALB} / \mathrm{c}$ mice are susceptible and develop fibrosis following FITC administration [56]. The disadvantages of this model include profound variability due to differences between batches of FITC, as well as in the method used to prepare the solution before instillation. Importantly, given the characteristics of the etiologic agent used to induce this model of IPF, this model is considered a very artificial system with limited clinical relevance [56].

Adenovirus vectors have been used to overexpress the pro-fibrotic cytokine transforming growth factor (TGF)- $\beta$, which results in pulmonary fibrosis. As TGF- $\beta$ overexpression in the lungs is known to be crucial in the development of fibrosis in humans [62], this model mimics an important feature of disease etiology. However, the delivery system has some drawbacks, as the virus itself initiates an immune response. Moreover, adenoviruses display 
significant tropism for epithelial cells and rarely infect other cell types such as fibroblasts [58], which are the cells meant to be targeted in this model. As TGF- $\beta$ has major effects on fibroblast biology, the main feature of this model is the effect of epitheliumderived TGF- $\beta$ on fibroblasts and myofibroblasts, resulting in the deposition of ECM proteins and areas of dense fibrosis [63]. An advantage of this model is the relatively low degree of inflammation, as well as what appears to be a direct effect on fibroblasts/ myofibroblasts [63], which is in accordance with the clinical situation (as we understand it today).

6.5 Silica

Silica administration induces a similar pathology in mouse lungs as in humans exposed to silica, and as is also observed in human silicainduced fibrosis, structural remodeling persists when administration is halted [56]. Following the administration of silica particles, fibrotic nodules develop in mouse lungs, with considerable resemblance to the human lesions that develop after exposure to mineral fibers [56]. The fibrotic response is accompanied by a limited inflammatory response, and different pro-fibrotic cytokines such as TGF- $\beta$, platelet-derived growth factor, and IL-10 are involved in disease development, which is in accordance with the clinical situation [56]. Another advantage is that nodules develop around silica fibers, and these fibers are easy to identify by light microscopy. The response in this model is strain-dependent, with C57BL/6 mice being the most susceptible. The main drawbacks are the time required to establish disease, i.e., 30-60 days, and the need for special equipment to aerosolize the silica particles. However, since the route of administration, the driving etiologic agent, and the resulting pathobiology are all similar to the characteristics of this subtype of pulmonary fibrosis $[56,58]$, the silica exposure model can be considered to have very good clinical relevance.

\section{What Does the Future Hold for Mouse Models of Human Disease?}

Medical research using experimental animals (not only mice but other animals including rats, guinea pigs, zebrafish, and fruit flies) has greatly contributed to many important scientific and medical advances in the past century and will continue to do so into the near future. These advances have contributed to the development of new medicines and treatments for human disease and have therefore played a vital role in increasing the human life span and improving quality of life.

Despite the acknowledged benefits of performing research using experimental animals, a number of considerations must be made before embarking on this type of research. Of course, the financial aspects of conducting this type of work are an important limitation, as the costs of purchasing and housing mice can be prohibitive, especially when genetically modified mice and colony 
maintenance are required for the study. The practicalities of working with animals such as mice may also be an issue, as this type of work requires specialized facilities, equipment, and staff to ensure studies are carried out in a manner that is safe for both the researchers and the animals. Moreover, as discussed in detail in this chapter, the relevance of the selected animal model to human disease must be carefully evaluated to ensure that these experiments provide robust results that are translatable to human health and disease. Another important and demanding aspect of biomedical research using animals is the ethics of imposing pain and suffering on live animals.

Although there has been a considerable reduction in the numbers of animals used in research in the last 30 years, animal research remains a vital part of biomedical research. However, no responsible scientist wants to cause unnecessary suffering in experimental animals if it can be avoided, so scientists have accepted controls on the use of animals for medical research. In the UK, this ethical framework has been enshrined in law, i.e., the Animals (Scientific Procedures) Act 1986. This legislation requires that applications for a project license to perform research involving the use of "protected" animals (including all vertebrates and cephalopods) must be fully assessed with regard to any harm imposed on the animals. This involves a detailed examination of the proposed procedures and experiments, and the numbers and types of animal used, with robust statistical calculations to support these numbers. The planned studies are then considered in light of the potential benefits of the project. Both within and outside the UK, approval for a study involving protected animals also requires an internal ethical review process, usually conducted by the research institution where the work is taking place, with the aim of promoting animal welfare by ensuring the work will be carried out in an ethical manner and that the use of animals is justified. Additionally, the UK has a national animal use reduction strategy supported by the National Centre for the Replacement, Refinement and Reduction of Animals in Research (NC3Rs; London, UK). This consortium was established in 2004 to promote and develop high-quality research that takes the principles of replacement, refinement, and reduction (the 3Rs) into account.

\subsection{Replacement}

Replacement strategies often involve the use of alternative, non-protected species (e.g., zebrafish, fruit flies, flatworms) and in vitro correlates (two-dimensional cell culture or threedimensional organoids containing multiple cell types) to test hypotheses and assess the effects of therapeutic interventions. The main obstacle with studies on non-protected animals is the difficulty of accurately mimicking the complex physiological systems involved in human health and disease, as described in detail above. For example, the fruit fly Drosophila melanogaster is an excellent 
model organism for studies on genetic diseases, aging, and pathogen-borne illnesses but may be less relevant for studies on complex lung diseases. Importantly, model organisms such as fruit flies, zebrafish, and flatworms do not possess lungs, which somewhat limits the translatability of research on these animals in the field of respiratory disease. As such, it is likely that rodents will remain the model organism of choice for studies into lung disease for some time to come.

There has been considerable progress recently in imitating single organs such as the liver, lung, and brain in vitro using multiple cell types and a physical scaffold. As an important advantage, these in vitro tests have replaced a large number of rodents in initial drug discovery experiments, while also speeding up the process [64]. These studies still require further refinement and validation to establish them as suitable models for an entire organ; importantly, these in vitro organoids cannot take into account interactions between organ systems in complex, multisystem diseases such as COPD.

\subsection{Refinement}

Refinement involves selecting the most clinically relevant model for the disease available, informed by the discussion above on closely recapitulating the etiologic agent and disease pathobiology associated with clinical cases. Another important factor is refining the management of pain. An assessment of the procedures used and the effects of the substance on the animal, as well as the degree of handling, restraint, and analgesia, are other important aspects of refinement. This standard of animal care is achieved through strict regulations and controls on how personnel are trained to carry out experiments on live animals. Adequate training is an important aspect of refinement and should be reviewed and improved on an ongoing basis. Moreover, refinement can be achieved by improving animal housing by environmental enrichment, e.g., providing a place for mice to hide in the cage and housing social animals such as mice in appropriate-sized groups. These simple changes can improve the physiological and behavioral status of research animals; this not only increases animal well-being but also contributes to the quality of the experimental results by reducing stress levels.

\subsection{Reduction}

The 3Rs aspect of reduction focuses on the statistical power of experiments and by following the Animal Research: Reporting of In Vivo Experiments (ARRIVE) guidelines, originally published in PLOS Biology in 2010. These guidelines provide a framework to improve the reporting of research performed on live animals by maximizing the quality of the scientific data and by minimizing unnecessary studies. The ARRIVE guidelines provide a checklist of aspects that must be considered in good quality research using live animals. The guidelines are most appropriate for comparative studies involving two or more groups of experimental animals with at least one control group, but they also apply to studies involving 
drug dosing in which a single animal is used as its own control (within-subject experiments). The guidelines provide recommendations on what should be considered when preparing to report on the results of experiments involving live animals, i.e., by providing a concise but thorough background on the scientific theory and why and how animals were used to test a hypothesis, a statement on ethical approvals and study design including power and sample size calculations, a clear description of the methods used to ensure repeatability, objective measurements of outcomes and adverse effects, and interpretation of the results in light of the available literature and the limitations of the study. In addition to the positive impact of the ARRIVE guidelines on reducing the number of animals used in experiments, this checklist provides an easy-tofollow roadmap on what is required for good quality reporting of experimental results.

\section{Conclusion}

In conclusion, the use of animals in research will continue to be an important aspect of medical research, and these procedures can be ethically justified provided the proper controls are in place. The benefits of animal research have been vital to the progress of medical science; abandoning these studies would have severe negative consequences on human health. By considering aspects such as the 3Rs and the ARRIVE guidelines in planning experiments involving live animals, the number of animals used and suffering of these animals for the benefit of human health can be minimized. This requires a strong regulatory framework such as that found in the UK and many other countries, as well an ongoing public debate on the advantages and limitations of animal experimentation.

\section{References}

1. Vandenbergh J (2000) Use of house mice in biomedical research. ILAR J 41:133-135

2. Hedrich H (ed) (2012) The laboratory mouse. Academic Press, Cambridge

3. Yue F, Cheng Y, Breschi A, Vierstra J, Wu W et al (2014) A comparative encyclopedia of DNA elements in the mouse genome. Nature 515:355-364

4. Cheng Y, Ma Z, Kim BH, Wu W, Cayting P et al (2014) Principles of regulatory information conservation between mouse and human. Nature 515:371-375

5. Bodenreider O, Hayamizu TF, Ringwald M, De Coronado S, Zhang S (2005) Of mice and men: aligning mouse and human anatomies. AMIA Annu Symp Proc 2005:61-65
6. Perlman RL (2016) Mouse models of human disease: an evolutionary perspective. Evol Med Public Health 2016(1):170-176

7. Townsley MI (2012) Structure and composition of pulmonary arteries, capillaries, and veins. Compr Physiol 2:675-709

8. Mitzner W, Lee W, Georgakopoulos D, Wagner E (2000) Angiogenesis in the mouse lung. Am J Pathol 157:93-101

9. Yuan X, Zhang J, Ao G, Quan C, Tian Y, Li H (2012) Lung cancer perfusion: can we measure pulmonary and bronchial circulation simultaneously? Eur Radiol 22:1665-1671

10. Flajnik MF, Kasahara M (2010) Origin and evolution of the adaptive immune system: genetic events and selective pressures. Nat Rev Genet 11:47-59 
11. Bailey M, Christoforidou Z, Lewis MC (2013) The evolutionary basis for differences between the immune systems of man, mouse, pig and ruminants. Vet Immunol Immunopathol 152:13-19

12. Mestas J, Hughes CC (2004) Of mice and not men: differences between mouse and human immunology. J Immunol 172:2731-2738

13. Doeing DC, Borowicz JL, Crockett ET (2003) Gender dimorphism in differential peripheral blood leukocyte counts in mice using cardiac, tail, foot, and saphenous vein puncture methods. BMC Clin Pathol 3:3

14. Choo JK, Seebach JD, Nickeleit V, Shimizu A, Lei H, Sachs DH, Madsen JC (1997) Species differences in the expression of major histocompatibility complex class II antigens on coronary artery endothelium: implications for cellmediated xenoreactivity. Transplantation 64:1315-1322

15. McAdam AJ, Greenwald RJ, Levin MA, Chernova T, Malenkovich $\mathrm{N}$ et al (2001) ICOS is critical for CD40-mediated antibody class switching. Nature 409:102-105

16. Grimbacher B, Hutloff A, Schlesier M, Glocker E, Warnatz K et al (2003) Homozygous loss of ICOS is associated with adultonset common variable immunodeficiency. Nat Immunol 4:261-268

17. Chapoval AI, Ni J, Lau JS, Wilcox RA, Flies DB et al (2001) B7-H3: a costimulatory molecule for $\mathrm{T}$ cell activation and IFN-gamma production. Nat Immunol 2:269-274

18. Zhang Y, Fear DJ, Willis-Owen SA, Cookson WO, Moffatt MF (2016) Global gene regulation during activation of immunoglobulin class switching in human B cells. Sci Rep 6:37988

19. Farrar JD, Smith JD, Murphy TL, Leung S, Stark GR, Murphy KM (2000) Selective loss of type I interferon-induced STAT4 activation caused by a minisatellite insertion in mouse Stat2. Nat Immunol 1:65-69

20. Schmitt N, Ueno H (2015) Regulation of human helper $\mathrm{T}$ cell subset differentiation by cytokines. Curr Opin Immunol 34:130-136

21. Pearce EJ, Sher A (1991) Functional dichotomy in the CD4+ $\mathrm{T}$ cell response to Schistosoma mansoni. Exp Parasitol 73:110-116

22. Dharmadhikari AS, Nardell EA (2008) What animal models teach humans about tuberculosis. Am J Respir Cell Mol Biol 39:503-508

23. Jang Y, Gerbec ZJ, Won T, Choi B, Podsiad A, BM B, Malarkannan S, Laouar Y (2018) Cutting edge: check your mice-a point mutation in the Ncrl locus identified in CD45.1 congenic mice with consequences in mouse susceptibility to infection. J Immunol 200:1982-1987
24. Calado RT, Dumitriu B (2013) Telomere dynamics in mice and humans. Semin Hematol 50:165-174

25. Ernst M, Abu Dawud R, Kurtz A, Schotta G, Taher L, Fuellen G (2015) Comparative computational analysis of pluripotency in human and mouse stem cells. Sci Rep 5:7927

26. Macieira-Coelho A, Azzarone B (1988) The transition from primary culture to spontaneous immortalization in mouse fibroblast populations. Anticancer Res 8:669-676

27. Williams K, Roman J (2016) Studying human respiratory disease in animals - role of induced and naturally occurring models. J Pathol 238:220-232

28. Bischoff SC (2007) Role of mast cells in allergic and non-allergic immune responses: comparison of human and murine data. Nat Rev Immunol 7:93-104

29. Schmit D, Le DD, Heck S, Bischoff M, Tschernig $\mathrm{T}$ et al (2017) Allergic airway inflammation induces migration of mast cell populations into the mouse airway. Cell Tissue Res 369:331-340

30. Andersson CK, Mori M, Bjermer L, Lofdahl CG, Erjefalt JS (2009) Novel site-specific mast cell subpopulations in the human lung. Thorax 64:297-305

31. Mullane K, Williams M (2014) Animal models of asthma: reprise or reboot? Biochem Pharmacol 87:131-139

32. Holgate ST, Wenzel S, Postma DS, Weiss ST, Renz H, Sly PD (2015) Asthma. Nat Rev Dis Primers 1:15025

33. Ray A, Oriss TB, Wenzel SE (2015) Emerging molecular phenotypes of asthma. Am J Physiol Lung Cell Mol Physiol 308:L130-L140

34. Mueller RS, Janda J, Jensen-Jarolim E, Rhyner C, Marti E (2016) Allergens in veterinary medicine. Allergy 71:27-35

35. O'Brien R, Ooi MA, Clarke AH, Thomas WR (1996) Immunologic responses following respiratory sensitization to house dust mite allergens in mice. Immunol Cell Biol 74:174-179

36. Shin YS, Takeda K, Gelfand EW (2009) Understanding asthma using animal models. Allergy Asthma Immunol Res 1:10-18

37. Durham CG, Schwiebert LM, Lorenz RG (2013) Use of the cockroach antigen model of acute asthma to determine the immunomodulatory role of early exposure to gastrointestinal infection. Methods Mol Biol 1032:271-286

38. Islam SA, Luster AD (2012) T cell homing to epithelial barriers in allergic disease. Nat Med 18:705-715 
39. Rydell-Tormanen K, Uller L, Erjefalt JS (2009) Allergic airway inflammation initiates long-term vascular remodeling of the pulmonary circulation. Int Arch Allergy Immunol 149:251-258

40. Calderon MA, Linneberg A, Kleine-Tebbe J, De Blay F, Hernandez Fernandez de Rojas D, Virchow JC, Demoly P (2015) Respiratory allergy caused by house dust mites: what do we really know? J Allergy Clin Immunol 136:38-48

41. Johnson JR, Wiley RE, Fattouh R, Swirski FK, Gajewska BU et al (2004) Continuous exposure to house dust mite elicits chronic airway inflammation and structural remodeling. Am J Respir Crit Care Med 169:378-385

42. Rydell-Tormanen K, Johnson JR, Fattouh R, Jordana M, Erjefalt JS (2008) Induction of vascular remodeling in the lung by chronic house dust mite exposure. Am J Respir Cell Mol Biol 39:61-67

43. Doras C, Petak F, Bayat S, Baudat A, Von Garnier C, Eigenmann P, Habre W (2017) Lung responses in murine models of experimental asthma: value of house dust mite over ovalbumin sensitization. Respir Physiol Neurobiol 247:43-51

44. Sarpong SB, Zhang LY, Kleeberger SR (2003) A novel mouse model of experimental asthma. Int Arch Allergy Immunol 132:346-354

45. Campbell EM, Kunkel SL, Strieter RM, Lukacs NW (1998) Temporal role of chemokines in a murine model of cockroach allergen-induced airway hyperreactivity and eosinophilia. J Immunol 161:7047-7053

46. Kurup VP, Grunig G (2002) Animal models of allergic bronchopulmonary aspergillosis. Mycopathologia 153:165-177

47. Templeton SP, Buskirk AD, Green BJ, Beezhold DH, Schmechel D (2010) Murine models of airway fungal exposure and allergic sensitization. Med Mycol 48:217-228

48. Kumar RK, Herbert C, Foster PS (2016) Mouse models of acute exacerbations of allergic asthma. Respirology 21:842-849

49. Maltby S, Tay HL, Yang M, Foster PS (2017) Mouse models of severe asthma: understanding the mechanisms of steroid resistance, tissue remodelling and disease exacerbation. Respirology 22:874-885

50. Bashir A, Shah NN, Hazari YM, Habib M, Bashir $S$ et al (2016) Novel variants of SERPINlA gene: interplay between alphalantitrypsin deficiency and chronic obstructive pulmonary disease. Respir Med 117:139-149

51. Groneberg DA, Chung KF (2004) Models of chronic obstructive pulmonary disease. Respir Res 5:18
52. Bartalesi B, Cavarra E, Fineschi S, Lucattelli M, Lunghi B et al (2005) Different lung responses to cigarette smoke in two strains of mice sensitive to oxidants. Eur Respir J 25:15-22

53. D'Armiento J, Dalal SS, Okada Y, Berg RA, Chada K (1992) Collagenase expression in the lungs of transgenic mice causes pulmonary emphysema. Cell 71:955-961

54. Knudsen L, Ruppert C, Ochs M (2017) Tissue remodelling in pulmonary fibrosis. Cell Tissue Res 36:607-626

55. Martinez FJ, Collard HR, Pardo A, Raghu G, Richeldi L et al (2017) Idiopathic pulmonary fibrosis. Nat Rev Dis Primers 3:17074

56. Tashiro J, Rubio GA, Limper AH, Williams K, Elliot SJ et al (2017) Exploring animal models that resemble idiopathic pulmonary fibrosis. Front Med (Lausanne) 4:118

57. Walkin L, Herrick SE, Summers A, Brenchley PE, Hoff CM et al (2013) The role of mouse strain differences in the susceptibility to fibrosis: a systematic review. Fibrogenesis Tissue Repair 6:18

58. Moore BB, Hogaboam CM (2008) Murine models of pulmonary fibrosis. Am J Physiol Lung Cell Mol Physiol 294:L152-L160

59. Manitsopoulos N, Nikitopoulou I, Maniatis NA, Magkou C, Kotanidou A, Orfanos SE (2018) Highly selective endothelin-1 receptor A inhibition prevents bleomycin-induced pulmonary inflammation and fibrosis in mice. Respiration 95:122-136

60. Yatomi M, Hisada T, Ishizuka T, Koga Y, Ono A et al (2015) 17(R)-resolvin Dl ameliorates bleomycin-induced pulmonary fibrosis in mice. Physiol Rep 3:e12628

61. Rydell-Tormanen K, Andreasson K, Hesselstrand R, Risteli J, Heinegard D et al (2012) Extracellular matrix alterations and acute inflammation; developing in parallel during early induction of pulmonary fibrosis. Lab Investig 92:917-925

62. Gauldie J, Kolb M, Ask K, Martin G, Bonniaud P, Warburton D (2006) Smad3 signaling involved in pulmonary fibrosis and emphysema. Proc Am Thorac Soc 3:696-702

63. Sime PJ, Xing Z, Graham FL, Csaky KG, Gauldie J (1997) Adenovector-mediated gene transfer of active transforming growth factorbetal induces prolonged severe fibrosis in rat lung. J Clin Invest 100:768-776

64. Festing S, Wilkinson R (2007) The ethics of animal research. Talking Point on the use of animals in scientific research. EMBO Rep $8: 526-530$ 